\title{
Papers
}

\section{Paraffin wax embedded muscle is suitable for the diagnosis of muscular dystrophy}

\author{
I N Sheriffs, D Rampling, V V Smith
}

\begin{abstract}
Aim-At present, the diagnosis of muscular dystrophy is made by means of immunohistochemistry on frozen sections. The aim of this study was to develop a sensitive and reproducible immunohistochemical method for use on formalin fixed, paraffin wax embedded sections for the demonstration of dystrophin associated proteins and other muscle associated antigens.

Methods-All the cases studied were from the files of the department of histopathology, Great Ormond Street Hospital for Children NHS Trust. Immunohistochemistry was performed on paraffin wax embedded sections with heat mediated antigen retrieval and overnight incubation with the antibodies at room temperature. Four different pretreatment buffers were tested in the attempt to optimise the immunostaining. Frozen sections were run in parallel for direct comparison.

Results-All the antibodies except $\delta$ sarcoglycan gave strong, consistent immunostaining in paraffin wax embedded sections, comparable with the frozen sections. The most consistent results were obtained using citrate/EDTA as the pretreatment buffer.

Conclusion-A reliable and reproducible technique has been established, using a heat mediated citrate/EDTA buffer antigen retrieval method, which works well for most of the antibodies needed to make the diagnosis of muscular dystrophy in formalin fixed, paraffin wax embedded sections. This technique overcomes some of the inherent problems encountered using frozen muscle tissue and it could become a valuable tool for the diagnosis of muscular dystrophy.

(f Clin Pathol 2001;54:517-520)
\end{abstract}

Keywords: immunohistochemistry; muscle biopsies; muscular dystrophy; paraffin wax sections

Muscular dystrophy constitutes a complex group of diseases with wide ranging severities. The main forms are Duchenne muscular dystrophy (DMD), ${ }^{1}$ Becker muscular dystrophy (BMD), ${ }^{2}$ congenital muscular dystrophy (CMD), ${ }^{34}$ and limb girdle muscular dystrophy (LGMD). ${ }^{5}$
Muscular dystrophies are caused by genetic abnormalities that result in transcription errors of dystrophin or its associated proteins. Dystrophin is a muscle cytoskeletal protein closely associated with proteins on the muscle membrane forming a complex that links intracellular actin to extracellular matrix proteins such as merosin (laminin $\alpha 2$ ). The transmembrane complex is primarily composed of $\alpha, \beta, \delta$, and $\gamma$ sarcoglycans, and $\alpha$ and $\beta$ dystroglycans. Mutations in genes encoding these proteins give rise to the different forms of muscular dystrophy. Altered dystrophin expression causes DMD or BMD, ${ }^{6}$ a lack of merosin expression results in $\mathrm{CMD},{ }^{7}$ and abnormalities in the four sarcoglycans cause four distinct subtypes of LGMD. ${ }^{8}$

These proteins can be detected by western blotting or by immunohistochemistry; with the latter being thought to be the more sensitive technique. This is because of an apparent loss or breakdown of an epitope of dystrophin that occurs during processing for western blotting. ${ }^{9}$ It has also been suggested that immunohistochemical analysis is more reliable than gene analysis using the multiplex polymer chain reaction, which only reveals approximately $70 \%$ of DMD mutations. ${ }^{10}$ Immunohistochemistry has become a powerful tool in distinguishing different muscular dystrophies because of its speed, accuracy, and the increasing availability of antibodies to dystrophin and its associated proteins.

Muscular dystrophy is usually detected early in children and is diagnosed by a muscle biopsy. Histological examination of frozen sections shows a pronounced increase in connective tissue with haematoxylin and eosin as well as trichrome staining. The definitive diagnosis can be made by means of immunohistochemistry for dystrophin and its associated proteins. Antibodies against these antigens are commercially available but their technical specifications are for use on frozen sections only. Other antibodies commonly used in the diagnosis of muscle disease that are also said to be for use on frozen sections only are antibodies to myosin heavy chain, which are used in fibre typing.

The problems encountered when using frozen muscle biopsies include freezing, handling, safety, and storage. In addition, only having access to archival material, postmortem 
Table 1 Details of the antibodies used in our study

\begin{tabular}{llll}
\hline Antibody/antigen & Source & Clone & Dilution \\
\hline Dystrophin (rod domain), DYS1 & Novacastra & Dy4/6D3 & $1 / 10$ \\
Dystrophin (C-terminus), DYS2 & Novacastra & Dy8/6C5 & $1 / 30$ \\
Dystrophin (N-terminus), DYS3 & Novacastra & Dy10/12B2 & $1 / 30$ \\
Merosin M-chain & Chemicon & 5H2 & $1 / 7000$ \\
Spectrin & Novacastra & RBC2/3D5 & $1 / 100$ \\
$\alpha$ Sarcoglycan (adhalin) & Novacastra & AD1/20A6 & $1 / 400$ \\
$\beta$ Sarcoglycan & Novacastra & bSarc1/5B1 & $1 / 150$ \\
$\delta$ Sarcoglycan & Novacastra & dSarc3/12C1 & $1 / 20$ \\
$\gamma$ Sarcoglycan & Novacastra & 35DAG/21B5 & $1 / 800$ \\
$\beta$ Dystroglycan & Novacastra & 43DAG/8D5 & $1 / 200$ \\
Myosin heavy chain (fast) & Novacastra & WB-MHCf & $1 / 200$ \\
Myosin heavy chain (slow) & Novacastra & WB-MHCs & $1 / 100$ \\
& & &
\end{tabular}

tissue, or referred sections is an extra difficulty. These problems prompted us to develop a sensitive and reproducible immunohistochemical method on paraffin wax embedded sections for the demonstration of dystrophin, its associated proteins, and other muscle associated antigens.

\section{Materials and methods}

The specimens used in our study were from eight patients diagnosed at our institution. Five had muscular dystrophy, one had muscular dystrophy with type I fibre predominance, one had muscular dystrophy with type II fibre predominance, and one biopsy was within normal limits (this biopsy was used as a normal control). Frozen sections from each of the patients were run in parallel with paraffin wax embedded sections for comparison.

The tissues were fixed in $4 \%$ neutral buffered formalin for 24 hours, processed into paraffin wax, then $8 \mu \mathrm{m}$ thick sections were cut and placed on to silane coated slides (aminopropyltriethoxysilane; Sigma, Poole, Dorset, UK). The sections were dried overnight at $37^{\circ} \mathrm{C}$, dewaxed, and rehydrated. Endogenous peroxidase was blocked using 5\% hydrogen peroxide (Sigma) for 20 minutes and the sections were subjected to heat mediated antigen retrieval. Different pretreatment buffers were tested to optimise the immunostaining. These comprised citrate buffer (10 $\mathrm{mM}$ citric acid, pH 6.0), EDTA buffer (1 mM, pH 8.0), citrate/EDTA buffer (10 mM citric acid/ $2 \mathrm{mM}$ EDTA, pH 6.2), and boric acid buffer (20 mM, pH 7.0).

Pretreatment for all buffers was carried out in a Biomen pressure cooker inside a domestic microwave oven (Panasonic, $800 \mathrm{~W}$ ). The slides held in a plastic rack were placed into the boiling buffer and pressure cooked at maximum pressure for four minutes. The slides were then transferred to cold water before immunostaining.

The antibodies and concentrations used are listed in table 1 . They were diluted in $1 \%$ bovine serum albumen (Sigma) in phosphate buffered saline (PBS) and incubated overnight at room temperature in a humidity chamber. The immunoreaction was localised using the Duet detection system (Dako, Ely, Cambridgeshire, UK), which is a two step strepavidinbiotin-peroxidase method. Each layer was incubated for one hour at room temperature, with a three minute wash in $0.1 \%$ Tween 20 in PBS between each step. Visualisation was achieved using 3,3'-diaminobenzidine tetrachloride (DAB; Sigma). Sections were counterstained with Mayer's haematoxylin, dehydrated, cleared, and mounted in Styrolite (BDH, Poole, Dorset, UK).

For frozen section immunohistochemistry, muscle specimens were snap frozen in cold hexane $\left(-80^{\circ} \mathrm{C}\right)$, cryostat sections were cut ( $8 \mu \mathrm{m}$ thick) and dried on glass slides. No fixation or pretreatment was carried out before immunostaining. The immunoreaction was localised with biotinylated rabbit antimouse antibody followed by the avidin-biotinperoxidase complex method (Dako) and visualised with DAB.

For each run, a section omitting the primary antibody was included as a negative control and spectrin immunostaining was used as a marker of membrane integrity in each patient.

\section{Results}

In the control muscle specimen, identical immunostaining patterns were seen in both the paraffin wax and frozen sections with all but one of the antibodies tested (fig 1). Strong muscle membrane staining was detectable with the exception of the antibody to $\delta$ sarcoglycan which, despite being strongly positive on frozen sections, was consistently negative on paraffin wax embedded sections. Fixation and processing caused some shrinkage of muscle fibres; however, this made little difference to the immunostaining properties except for a slight sarcoplasmic staining, which did not affect interpretation. The citrate buffer pretreatment worked well; however, the citrate/EDTA buffer gave superior results for all the antibodies tested except anti- $\delta$ sarcoglycan. Sections immunostained after pretreatment with either the EDTA or boric acid buffers were negative for all of the antibodies tested. Therefore, the citrate/EDTA buffer was the preferred pretreatment for paraffin wax embedded muscle sections.

In the five patients with DMD and the two who showed fibre type predominance, immunostaining patterns were comparable in paraffin wax and frozen sections, thus allowing a definitive diagnosis to be made.

\section{Discussion}

We found that immunohistochemistry for dystrophin and its associated proteins worked well on formalin fixed, paraffin wax embedded sections after pressure cooking in citrate/EDTA buffer. This is an easy to follow method that produces consistent and reproducible results, which can be adapted by any histopathology laboratory. The ability to use paraffin wax sections rather than frozen sections in the study of muscular dystrophy makes this method valuable to a routine histopathology laboratory, as well as a more specialised unit. One of the main advantages is specimen storage. The use of paraffin wax embedded material overcomes the need for low temperature, high maintenance freezers, which are expensive both to buy and to run. Stored frozen muscle biopsies are prone to freeze drying, rendering them useless for future research or further disease classification. 

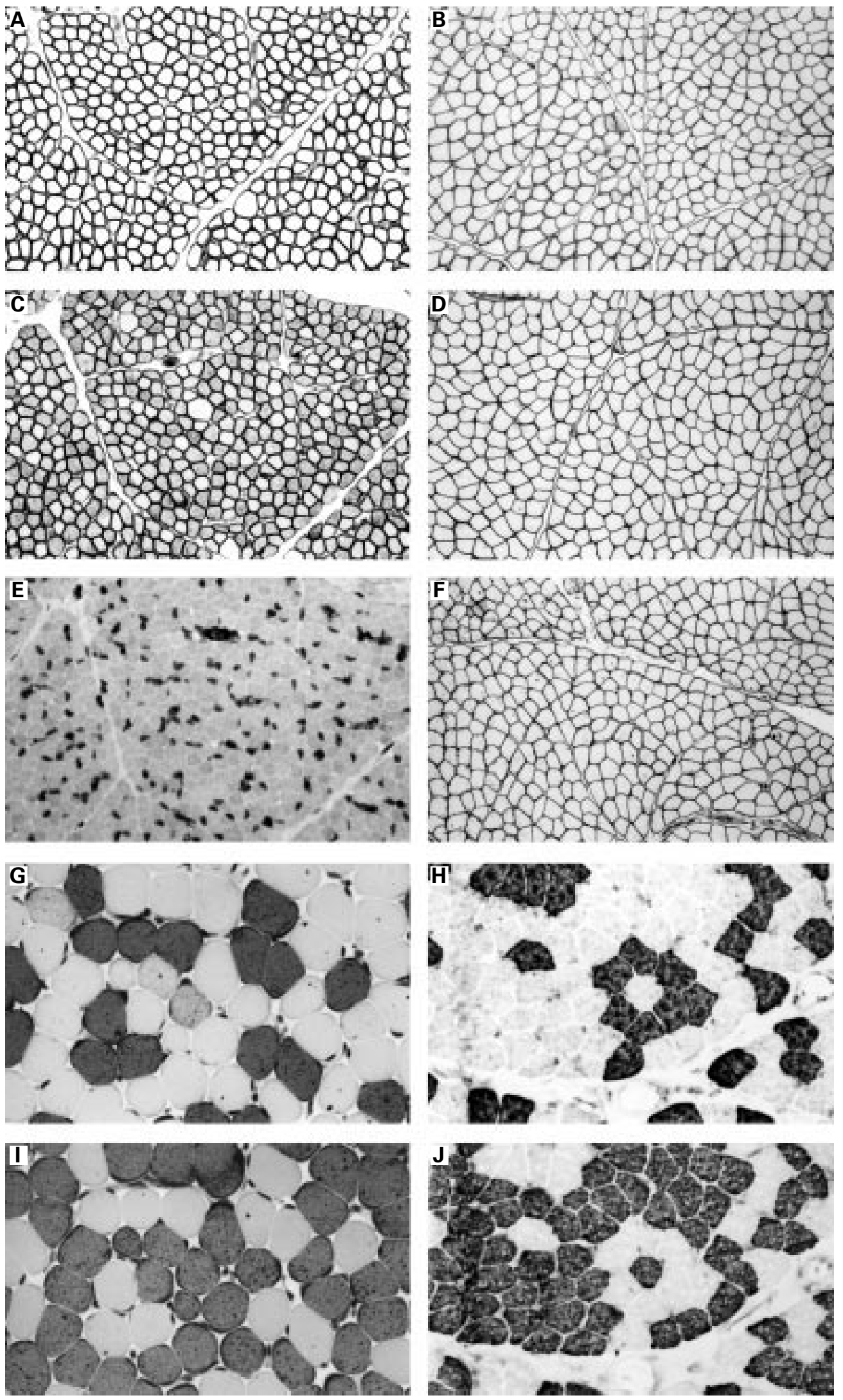

Figure 1 Comparison of paraffin wax embedded sections and frozen sections. Sections were immunostained with antibodies to dystrophin 2 ( $A$, paraffin wax section; $B$, frozen section), spectrin ( $C$, paraffin wax section; $D$, frozen section), $\delta$ sarcoglycan ( $E$, paraffin wax section; $F$, frozen section), fast myosin heavy chain ( $G$, paraffin wax section; $H$, frozen section), and slow myosin heavy chain (I, paraffin wax section; F, frozen section). 
With the recent increase in antibody availability there is a need to carry out retrospective studies on archival muscle biopsies, often with a view to genetic counselling. A large proportion of archival frozen muscle samples prove to be unsuitable.

There are considerable pitfalls inherent in the freezing of muscle biopsies. Adequate freezing is essential to avoid ice crystal artefacts disrupting the muscle fibre architecture and creating difficulties with interpretation. To avoid this, either liquid nitrogen or dry ice must be used, which are both hazardous to handle and necessitate certain safety procedures. The handling of unfixed muscle tissue is also a health and safety concern, particularly with the increase of Creutzfeldt-Jakob disease and human immunodeficiency virus infection. Formalin fixation goes some way to overcome this problem. Correct orientation of the biopsy is also essential for interpretation and this can be difficult and time consuming, especially for small needle biopsies. By using formalin fixed tissue, orientation can occur after fixation, rather than before freezing, and fixation renders handling of small pieces of tissue easier. Once a muscle biopsy is processed and embedded in paraffin wax, good quality sections are achieved and storage is no longer a problem.

Because greater demands are being made on theatre time, many biopsies are now taken out of normal working hours, which involves considerable organisation to acquire an adequate specimen. Using formalin fixed, paraffin wax embedded tissue would not only overcome this problem, but would also allow the wider use of both referred surgical material and tissue taken at necropsy.

However, the processing of muscle biopsies does cause shrinkage of the muscle fibres and the morphology differs slightly from that seen on frozen sections. There may be a learning curve in reporting these biopsies. Our study clearly shows that the immunohistochemical staining of dystrophin and its associated proteins is identical to that on frozen sections. In addition, formalin fixed, paraffin wax embedded sections can be superior to frozen sections when studying the nature of an inflammatory infiltrate or cytoskeletal proteins.

Despite testing multiple antibody dilutions as well as four antigen retrieval techniques, the anti- $\delta$ sarcoglycan antibody failed on paraffin wax sections. This is of some concern because it is used for the diagnosis of limb girdle muscular dystrophy $2 \mathrm{~F}$ (LGMD2F), which is an autosomal recessive LGMD subtype. ${ }^{11}$ However, LGMD2F is rare. A report by Fanin et al of patients with LGMD, using muscle immunohistochemistry, showed that $39 \%$ had a deficiency of $\alpha$ sarcoglycan, $22 \%$ of $\gamma$ sarcoglycan,
$11 \%$ of $\beta$ sarcoglycan, none of $\delta$ sarcoglycan, and $28 \%$ had no deficiency of sarcoglycans. ${ }^{12}$ It has been suggested that when a deficiency of $\delta$ sarcoglycan occurs, a total loss of all the sarcoglycans in the transmembrane complex is seen; therefore, only two of the four antibodies against sarcoglycans need to be used. ${ }^{13}$ However, in patients where LGMD is suspected a piece of muscle should be frozen so that $\delta$ sarcoglycan can be studied if needed.

In conclusion, we have established a reliable and reproducible technique that works well with most of the antibodies needed to make a diagnosis of muscular dystrophy in formalin fixed, paraffin wax embedded muscle biopsies. Thus, frozen muscle tissue is no longer necessary for the diagnosis of muscular dystrophy, with the exception of LGMD2F; however, until this can be overcome a piece of muscle should always be frozen. With the future introduction of new antibodies it may be possible to use this technique to diagnose all muscle pathology, therefore negating the need for frozen muscle tissue. We feel that this technique will help to overcome some of the inherent problems that occur with frozen sections and will be invaluable in assessing archival material. It could therefore become a valuable tool in a neuropathologist's repertoire.

This work was undertaken by Great Ormond Street Hospital for Children NHS Trust, which received a proportion of its funding from the NHS Executive; the views expressed in this publication are those of the authors and not necessarily those of the NHS Executive.

1 Duchenne G. De l'électrisation localisée et son application à la patholologie et à la thérapeutique, 2nd ed. Paris: Baillière, 1861

2 Becker P, Kiener F. Eine neue X-chromasomale muskeldystrophie. Archiv für Psychiatrie und Nervenkrankheiten 1955; 193:427.

3 Battern F. Three cases of myopathy, infantile type. Brain 1903;27:147-8

4 Battern F. Myositis fibrosa. BMf 1903;2:1333.

5 Walton JN, Nattrass FJ. On the classification, natural history and treatment of the myopathies. Brain 1954;77:169-231.

6 Kunkel L. The Wellcome lecture, 1988. Muscular dystrophy: a time of hope. Proc R Soc Lond B Biol Sci 1989; 237:1-9.

7 Tome F, Evangelista T, Leclerc A, et al. Congenital muscular dystrophy with merosin deficiency. C R Acad Sci III 1994;317:351-7.

8 Lim LE, Campbell KP. The sarcoglycan complex in limb-girdle muscular dystrophy. Curr Opin Neurol 1998;11: 443-52.

9 Nicholson L, Johnson M, Davison K, et al. Dystrophin or a "related protein" in Duchenne muscular dystrophy. Acta Neurol Scand 1992;86:8-14.

10 Ozawa E, Noguchi S, Mizuno Y, et al. From dystrophinopathy to sarcoglycanopathy: evolution of a concept of muscular dystrophy. Muscle Nerve 1998;21:421-38.

11 Bushby KMD, Beckmann JS. Report of the 30th and 31st ENMC international workshop. The limb-girdle muscular dystrophies and proposal for a new nomenclature. Neuromuscul Disord 1995;5:337-43.

12 Fanin M, Duggan DJ, Mostacciuolo ML, et al. Genetic epidemiology of muscular dystrophies resulting from sarcoglycan gene mutations. F Med Genet 1997;34:973-7.

13 Bushby KM. Making sense of the limb-girdle muscular dystrophies. Brain 1999;122:1403-20. 